\title{
Pacific
}

Journal of

Mathematics

\section{HARMONIC NETS IN METRIC SPACES}

JÜRGEN JOST AND LEONARD TODJIHOUNDE 


\title{
HARMONIC NETS IN METRIC SPACES
}

\author{
JÜRGEN JOST AND LEONARD TODJIHOUNDE
}

\begin{abstract}
We investigate harmonic maps from weighted graphs into metric spaces that locally admit unique centers of gravity, like Alexandrov spaces with upper curvature bounds. We prove an existence result by constructing an iterative geometric process that converges to such maps, called harmonic nets for short.
\end{abstract}

\section{Introduction}

This paper deals with harmonic maps from weighted graphs into metric spaces. Such maps can be considered as a generalization of geodesic lines in Riemannian manifolds. A geodesic, considered as a map $\gamma:[0,1] \rightarrow N$ from the unit interval to the Riemannian manifold $N$ and parametrized proportionally to arclength, is characterized by the property that for all sufficiently close $0 \leq a<b \leq 1$, the point $\gamma((a+b) / 2)$ is the unique midpoint of $\gamma(a)$ and $\gamma(b)$, that is,

$$
\gamma\left(\frac{a+b}{2}\right)=\operatorname{argmin}_{q \in N}\left(d^{2}(\gamma(a), q)+d^{2}(\gamma(b), q)\right) .
$$

This leads us to represent a geodesic as a string of points in $N$, each of which is the midpoint of its two neighbors. At the same time, this allows us flexible refinements: we can insert additional points as midpoints of consecutive ones already present. For that, it is useful to also consider the following slight generalization of (1):

$$
\gamma(t a+(1-t) b)=\operatorname{argmin}_{q \in N}\left(t d^{2}(\gamma(a), q)+(1-t) d^{2}(\gamma(b), q)\right),
$$

where $0<t<1$.

A midpoint is a center of gravity of two points. In a Riemannian manifold, such centers of gravity exist locally uniquely, that is, when the points whose center is to be constructed are sufficiently close. Globally, uniqueness need not be true. Therefore, we may need to localize in the image.

It is then clear how to conceptualize a harmonic map from a weighted graph into $N$. We simply require that the images of the nodes of the graph by appropriately weighted centers of gravity of their neighbors. Here, in order to localize in the image, we might need to refine the graph by subdividing edges.

MSC2000: 58E20, 53C43, 53C22.

Keywords: harmonic map, weighted graph, center of gravity, refinement. 
Harmonic maps from graphs into compact Riemannian manifolds were studied in [Kotani and Sunada 2001]. Our approach, however, naturally leads to a generalization to any metric space locally admitting unique centers of gravity — and this includes the important class of Alexandrov spaces with upper curvature bounds (see [Berestovskij and Nikolaev 1993] as a systematic reference).

Thus, we show the existence of harmonic maps from weighted graphs into such metric spaces. Such maps are called harmonic nets in short. (In the case of a space of nonpositive curvature in the sense of Alexandrov or Busemann, this result is contained in a general existence result for harmonic maps; see [Jost 1994; 1997].) The proof is not difficult. It is based on the iterative replacement of image points by the centers of gravity of the images of their neighbors, following the strategy described in [Jost 1998], together with suitable adaptive refinements to keep the constructions local. Here it is important that the domain, that is, our graph, can be treated as a one-dimensional object. While two dimensions represent a borderline case, in higher dimensions, general constructions of harmonic maps are only possible when the target space possesses nonpositive curvature. The reason is that the energy functional we are employing is quadratic, and therefore the scaling behavior is different in dimensions 1,2, and greater than 2. The essential features of our scheme are local uniqueness and the scaling property of our functions.

Our constructions possess certain similarities with some schemes employed in numerical analysis, like the standard difference scheme for the numerical solution of the Laplace equation or adaptive refinements in multigrid methods. A key conceptual feature of our approach is that we systematically exploit the local uniqueness of solutions and that we need to make explicit only the images of a discrete set of points, because then all other images are implicitly determined by that local uniqueness. Therefore, as in good numerical schemes, we never have to work out or store more information than needed.

\section{Geometric concepts}

Let $(N, d)$ be a complete metric space. For conciseness, we usually write $N$ in place of $(N, d)$, the metric $d$ being understood. We say that $N$ admits refinements if any $p, q \in N$ have a midpoint, that is, if there exists $m \in N$ such that

$$
d(m, p)=d(m, q)=\frac{1}{2} d(p, q) .
$$

Definition 2.1. Suppose that $N$ admits refinements. We define the radius $r(N)$ of unique refinement as the largest $r \in[0, \infty]$ with the property that for any two $p, q \in N$ with $d(p, q) \leq 2 r$, their refinement (midpoint) $m=m(p, q)$ is unique.

More generally, we say that $q \in N$ is a center of gravity of the finitely many points $p_{1}, \ldots, p_{n} \in N$ with positive weights $w_{1}, \ldots, w_{n}$ if 


$$
q=\operatorname{argmin}_{p} \sum_{j=1}^{n} w_{j}^{2} d^{2}\left(p, p_{j}\right) .
$$

We define the convexity radius $c(N)$ as the largest $c \in[0, \infty]$ with the property that whenever $p, p_{1}, \ldots, p_{n}$ are points in $N$ with $d\left(p, p_{i}\right) \leq c$ for $i=1, \ldots, n$, the center of gravity of $p_{1}, \ldots, p_{n}$ (with any positive weights) is unique.

Since a midpoint is a center of gravity, we obviously have $0 \leq c(N) \leq r(N)$.

Let $\Gamma$ be a finite weighted graph with vertex set $I$ and edge set $E$, where each $e \in E$ has a weight $w(e)>0$. We say that two vertices $i, j$ are neighbors if they are connected by an edge. Thus, for our purposes, a graph is a discrete set $I$ together with a symmetric neighborhood (adjacency) relation $\sim$ and (symmetric) weights $w(i, j)=w(e)$ for neighboring vertices $i, j$ connected by the edge $e$.

We define the refinement $\Gamma_{r}$ of $\Gamma$ as the graph with vertex set $I \cup E$ and adjacency relation defined as follows: $i \in I$ and $e \in E$ are neighbors if $i \in e$ in $\Gamma$, and there are no other pairs of neighbors in $\Gamma_{r}$. The weights are $w(i, e)=\sqrt{2} w(e)$, where $w(e)$ is the weight of the edge $e$ in $\Gamma$. Further refinements of $\Gamma_{r}$ are defined iteratively.

A map $f$ from $\Gamma$ to $N$ assigns to every $i \in I$ some point $p=f(i)$ in $N$. We define the energy of such a map $f: \Gamma \rightarrow N$ as

$$
E(f)=\sum_{i \in I} E_{i}(f), \quad \text { where } \quad E_{i}(f)=\sum_{j \in I ; i \sim j} w^{2}(i, j) d^{2}(f(i), f(j)) .
$$

In particular, for $i \sim j$,

$$
d^{2}(f(i), f(j)) \leq \frac{1}{w^{2}(i, j)} E_{i}(f) .
$$

We say that the map $f$ is harmonic if for all $i \in I, f(i)$ is a center of gravity of the points $f(j), j \sim i$, with weights $w_{j}=w^{2}(i, j)$.

\section{Characterization by angles in tangent cones}

The above concepts of refinement and center of gravity find their natural place in the context of Alexandrov's metric spaces. For a systematic development of this theory that we shall use in this section, see [Berestovskij and Nikolaev 1993].

These spaces enjoy particular properties when their (Alexandrov) curvature is bounded from above. It is part of the definition of such a space of curvature bounded from above that any two sufficiently close points can be joined by a shortest geodesic which then is in fact unique and depends continuously on these endpoints. (We may also parametrize it by arclength - and call it an arclength geodesic - if convenient.) Some of the general notions in the theory, however, do not need the assumption of an upper curvature bound. That assumption then is rather employed to derive geometric properties of the objects defined in the theory. 
An important concept here is the tangent cone of a metric space at a point. Let $(N, d)$ be a metric space and $\gamma_{1}, \gamma_{2}:[0, \varepsilon] \rightarrow(N, d)$ arclength geodesics emanating from a point $P \in N$. Consider points $Q \in \gamma_{1}, R \in \gamma_{2}$ different from $P$. An (upper) angle $\theta\left(\gamma_{1}, \gamma_{2}\right)$ between $\gamma_{1}$ and $\gamma_{2}$ is defined by

$$
\cos \theta\left(\gamma_{1}, \gamma_{2}\right):=\varlimsup_{Q, R \rightarrow P} \frac{d^{2}(P, Q)+d^{2}(P, R)-d^{2}(Q, R)}{2 d(P, R) d(P, Q)} .
$$

In a metric space whose curvature is bounded from above by a constant $K \geq 0$, we have the following characterization of the angle between $\gamma_{1}$ and $\gamma_{2}$ (see [Bridson and Haefliger 1999, II.1-II.3]):

$$
\cos \theta\left(\gamma_{1}, \gamma_{2}\right)=\lim _{s \rightarrow 0} \frac{d\left(P, \gamma_{2}(\varepsilon)\right)-d\left(\gamma_{1}(s), \gamma_{2}(\varepsilon)\right)}{s},
$$

provided in case $K>0$ that $\varepsilon$ is less than the diameter of the comparison model space of constant curvature $K$.

A geodesic curve $\gamma$ starting at a point $P \in N$ has a direction if $\theta(\gamma, \gamma)=0$ and two curves have the same direction if the angle between them is equal to zero. This is an equivalence relation on the space of curves starting from the same point $P \in N$ and the completion of the set of equivalence classes (endowed with the distance induced by the angle) is called the space of directions $\Omega_{P}(N)$ of $N$ at the point $P$. The tangent cone $T_{P} N$ of $(N, d)$ at a point $P \in N$ is the cone over the space of directions, that is, $\Omega_{P}(N) \times \mathbb{R}_{+}$with points in $\Omega_{P}(N) \times\{0\}$ identified together.

We will denote a tangent element by $[\gamma, x]$, where $\gamma \in \Omega_{P}(N), x \geq 0$ and elements $[\gamma, 0]$ are identified with the origin $O_{p}$ of $T_{P} N$.

The distance $d$ in $N$ induces on $T_{P} N$ a distance function $\tilde{d}_{P}$ defined by

$$
\tilde{d}_{P}^{2}\left(\left[\gamma_{1}, x_{1}\right],\left[\gamma_{2}, x_{2}\right]\right)=\left\{\begin{array}{cc}
x_{1}^{2}+x_{2}^{2}-2 x_{1} x_{2} \cos \theta\left(\gamma_{1}, \gamma_{2}\right) & \text { if } \theta\left(\gamma_{1}, \gamma_{2}\right)<\pi \\
x_{1}+x_{2} & \text { if } \theta\left(\gamma_{1}, \gamma_{2}\right) \geq \pi .
\end{array}\right.
$$

For those $[\gamma, x]$ admitting a unique geodesic from $P$ with direction $\gamma$ that can be extended up to distance $x$, we define the endpoint of that geodesic segment as the exponential image of $[\gamma, x]$. The inverse of this exponential map, the projection map from the subset of $N$ where it is defined to the tangent cone $T_{P} N$, is denoted by $\pi_{P}$. For simply connected, complete, nonpositively curved metric spaces, $\pi_{P}$ is defined everywhere, distance nonincreasing and distance preserving in the radial direction; see [Wang 2000].

The following important result was proved in [Nikolaev 1995]:

Lemma 3.1. Let $(N, d)$ a metric space of curvature at most $K$, where $K \geq 0$. The tangent cone at a point of $N$ is a space of nonpositive curvature in the sense of Alexandrov. 
Let $P, Q, R$ be points in $N$ and define $Q_{s} \equiv(1-s) P+s Q$ as the point on a distance-realizing geodesic joining $P$ and $Q$ at distance $s . d(P, Q)$ from $P$. We have the Taylor expansions

$$
\begin{aligned}
d^{2}\left(Q_{s}, R\right) & =d^{2}(P, R)-2 s d(P, R) \cos \theta_{P}(Q, R)+a(s), \\
\tilde{d}_{P}^{2}\left(\pi_{P}\left(Q_{s}\right), \pi_{P}(R)\right) & =d^{2}(P, R)-2 s d(P, R) \cos \theta_{P}(Q, R)+b(s),
\end{aligned}
$$

in which $\lim _{s \rightarrow 0} a(s) / s=0$ and $\lim _{s \rightarrow 0} b(s) / s=0$. Here $\theta_{P}(Q, R)$ denotes the angle subtended between $Q$ and $R$ at $P$.

Proposition 3.2 [Izeki and Nayatani 2005; Wang 2000]. Let $f: \Gamma \rightarrow(N, d)$ be a harmonic map.

(i) For any $i \in I$, the point $\pi_{f(i)}(f(i))$ minimizes

$$
\sum_{j \sim i} w^{2}(i, j) \tilde{d}_{f(i)}^{2}\left(\cdot, \pi_{f(i)} f(j)\right) \quad \text { in } T_{f(i)} N .
$$

(ii) For any $i \in I$ and any $V \in T_{f(i)} N$ we have

$$
\sum_{j \sim i} w^{2}(i, j)\left\langle V, \pi_{f(i)} f(j)\right\rangle \leq 0,
$$

where $\langle$,$\rangle denotes the inner product defined on T_{f(i)} N$ by

$$
\left\langle\left[\gamma_{1}, x_{1}\right],\left[\gamma_{2}, x_{2}\right]\right\rangle=x_{1} x_{2} \cos \theta\left(\gamma_{1}, \gamma_{2}\right) .
$$

(iii) For any $i \in I$, the center of gravity in $T_{f(i)}$ of the points $\left(\pi_{f(i)} f(j)\right)_{j \sim i}$ with weights

$$
\left(\frac{w^{2}(i, j)}{w(i)}\right)_{j \sim i}
$$

coincides with the origin $O_{i}:=\pi_{f(i)} f(i)$ of $T_{f(i)} N$, where $w(i)=\sum_{j \sim i} w^{2}(i, j)$.

Inequality (ii) will be interpreted as the critical condition for harmonic nets.

\section{Refining maps}

If $N$ admits refinements, we can construct a refinement $f_{r}: \Gamma_{r} \rightarrow N$ of a map $f: \Gamma \rightarrow N$ by assigning to every edge $e$ connecting $i$ and $j$ in $\Gamma$ some midpoint of $f(i)$ and $f(j)$. We observe that for each $i \in \Gamma$, we have

$$
E_{i}\left(f_{r}\right)=\frac{1}{2} E_{i}(f)
$$

where on the left hand side $i$ is considered as an element of $\Gamma_{r}$. Also, by symmetry,

$$
\sum_{i \in I} E_{i}\left(f_{r}\right)=\sum_{e \in E} E_{e}\left(f_{r}\right)=\frac{1}{2} E\left(f_{r}\right),
$$

where we consider the $i$ 's and $e$ 's as vertices of $\Gamma_{r}$. From (3) and (4) we obtain: 
Lemma 4.1. If $f_{r}: \Gamma_{r} \rightarrow N$ is a refinement of $f: \Gamma \rightarrow N$, then $E\left(f_{r}\right)=E(f)$. If $f$ is harmonic, so is its refinement.

The converse holds when distances between images are sufficiently small, that is, when midpoints between images of neighbors are unique.

From (2) and Lemma 4.1, we conclude that by performing sufficiently many successive refinements, we may assume that all distances between the images of any two neighboring vertices are smaller than some prescribed $\epsilon>0$, for example smaller than $r(N)$ or $c(N)$ when that quantity is positive.

\section{Homotopy classes}

For the present purposes, we write $r(f)$ and $r(\Gamma)$ instead of $f_{r}$ and $\Gamma_{r}$ because we wish to consider the refinement as an operation that can be iterated. For example, $r^{2}(\Gamma)=\left(\Gamma_{r}\right)_{r}$ is obtained as the refinement of $\Gamma_{r}$. A refinable map $f: \Gamma \rightarrow N$ is then considered as a collection of iteratively refined maps $r^{n}(f): r^{n}(\Gamma) \rightarrow N$ for $n \in \mathbb{N}$.

Now assume that the refinement radius $r(N)$ is positive. We say that two maps $f_{1}, f_{2}: \Gamma \rightarrow N$ are geodesically close if for every $i \in \Gamma$ the distance $d\left(f_{1}(i), f_{2}(i)\right)$ is at most $2 r(N)$; that is, if the images of $i$ under $f_{1}$ and $f_{2}$ have a unique midpoint. A refinement of the pair $f_{1}, f_{2}$ is then defined to be the triple $f_{1}, f_{1,2}, f_{2}$, where

$$
f_{1,2}(i) \text { is the midpoint of } f_{1}(i) \text { and } f_{2}(i) \text { for every } i \in \Gamma \text {. }
$$

Two refinable maps $f, g: \Gamma \rightarrow N$ are geometrically homotopic if there exist refinable maps $f_{0}=f, f_{1}, f_{2}, \ldots, f_{A}=g$ (where $A \in \mathbb{N}$ ) such that $r^{n}\left(f_{j-1}\right)$ and $r^{n}\left(f_{j}\right)$ are geodesically close for any $n \in \mathbb{N}$ and any $1 \leq j \leq A$. This finite sequence can again be refined by putting in midpoint maps between consecutive sequence elements. Geometric homotopy is obviously an equivalence relation.

\section{Construction of harmonic nets}

We assume that $N$ admits centers of gravity. By subdividing suitable edges of $\Gamma$ as above, we may assume that $\Gamma$ is bipartite, that is, its vertex set is a disjoint union $I=I_{1} \cup I_{2}$ such that all the neighbors of any point in one of those subsets are contained in the other one. On the space $C=C(\Gamma, N)$ of maps $f: \Gamma \rightarrow N$, we define maps $\rho_{\alpha}: C \rightarrow C, \alpha=1,2$ with $\rho_{\alpha}(f)$ being the map obtained from $f: \Gamma \rightarrow N$ by replacing the image of every $f(i)$ for $i \in I_{\alpha}$ by a center of gravity of the $f(j)$ for $j \sim i$. As long as the centers of gravity are not unique, we need to make choices here, but in the situation where $c(N)>0$, we can assume that $\Gamma$ has been sufficiently refined (depending on an upper bound $E$ for the energy of $f$ ) so that the images $f(j)$ of the neighbors of any $i \in \Gamma$ possess a unique center of gravity. (This follows from (2) and the fact that the edge weights get multiplied 
by a factor of $\sqrt{2}$, that is, become larger, under each refinement.) In that case, the maps $\rho_{\alpha}(f)$ are unambiguously defined for all $f$ with $E(f) \leq E$.

The function $\rho_{\alpha}$ decreases (or rather, does not increase) the energy density $E_{i}(f)$ for points $i \in I_{\alpha}$, but not necessarily for those in the complement of $I_{\alpha}$. Nevertheless, since by symmetry $\sum_{i \in I_{1}} E_{i}(f)=\sum_{i \in I_{2}} E_{i}(f)=\frac{1}{2} E(f)$ (see (4)), we have:

Lemma 6.1. We have $E\left(\rho_{\alpha}(f)\right) \leq E(f)$ for all $f$.

Lemma 6.2. We have $E\left(\rho_{2}\left(\rho_{1}(f)\right)\right)=E(f)$ if and only if $f$ is harmonic.

Theorem 6.3. Let $(N, d)$ be a compact metric space that admits centers of gravity. Let $\Gamma$ be a finite weighted graph. Then, for any map $f: \Gamma \rightarrow N$, the iterations $f_{n}:=\left(\rho_{2} \rho_{1}\right)^{n} f$ contain a subsequence converging to a harmonic map.

Proof. Since $N$ is compact, we can find some sequence $v(n)$ of positive integers going to infinity for which $f_{v(n)}(i)$ converges to some point $f_{0}(i) \in N$ for every vertex $i$ of the finite graph $\Gamma$. We have

$$
f_{v(n+1)}=\left(\rho_{2} \rho_{1}\right)^{\mu(n)} f_{v(n)} \quad \text { for some } \mu(n) \in \mathbb{N} .
$$

Since the metric $d$ behaves continuously under convergence (since it defines the topology of $N)$, we have $E\left(f_{0}\right)=\lim _{n \rightarrow \infty} E\left(f_{v(n)}\right)$. At the same time,

$$
\lim E\left(f_{v(n+1)}\right)=\lim E\left(\left(\rho_{2} \rho_{1}\right)^{\mu(n)} f_{v(n)}\right) \leq \lim E\left(f_{v(n)}\right)=E\left(f_{0}\right),
$$

the inequality coming from Lemma 6.1 because $\mu(n) \geq 1$. Thus, equality has to hold throughout. Moreover, $\rho_{2} \rho_{1} f_{v(n)}$ converges to $\rho_{2} \rho_{1} f_{0}$, and so

$$
\begin{aligned}
E\left(\rho_{2} \rho_{1} f_{0}\right) & =\lim E\left(\left(\rho_{2} \rho_{1}\right)^{\mu(n)+1} f_{v(n)}\right) & & \text { (as before) } \\
& =E\left(f_{0}\right) & & \text { (from the preceding observation.) }
\end{aligned}
$$

Lemma 6.2 then implies that $f_{0}$ is harmonic.

The assumption of the theorem that the space $N$ admits centers of gravity is satisfied when $N$ has an upper curvature bound. For $k \in \mathbb{R}$, we denote by $D_{k}$ the diameter of the $n$-dimensional, complete, simply connected model space with constant sectional curvature $k$. We then have, from Alexandrov theory:

Lemma 6.4 [Berestovskij and Nikolaev 1993]. Let X be an Alexandrov space with curvature bounded above by $k$. For every $x \in X$, there exists a positive number $R_{x} \in\left(0, \frac{1}{2} D_{k}\right]$ such that the closed metric ball of radius $R_{x}$ centered at $x$ is a convex subset in $X$.

Remark. When $N$ has nonpositive curvature in the sense of Alexandrov or Busemann, our theorem is contained in a general theorem from [Jost 1994], and when $N$ is a compact Riemannian manifold, it follows from the fact that any homotopy class contains at least one harmonic map [Kotani and Sunada 2001]. 
Since distances of neighboring image points are controlled by the energy of a map - see (2) — we see that if the refinement radius $r(N)$ is positive, we may control the geometric homotopy class by assuming an energy bound and sufficiently refining the graph $\Gamma$.

\section{References}

[Berestovskij and Nikolaev 1993] V. N. Berestovskij and I. G. Nikolaev, "Multidimensional generalized Riemannian spaces", pp. 165-250 in Geometry IV, edited by Y. G.Reshetnyak, Encyclopaedia Math. Sci. 70, Springer, Berlin, 1993. MR MR1263965

[Bridson and Haefliger 1999] M. R. Bridson and A. Haefliger, Metric spaces of non-positive curvature, Grundlehren der Math. Wissenschaften 319, Springer, Berlin, 1999. MR 2000k:53038 Zbl 0988.53001

[Izeki and Nayatani 2005] H. Izeki and S. Nayatani, "Combinatorial harmonic maps and discretegroup actions on Hadamard spaces", Geom. Dedicata 114 (2005), 147-188. MR 2006k:58024 Zbl 02227558

[Jost 1994] J. Jost, "Equilibrium maps between metric spaces", Calc. Var. Partial Differential Equations 2:2 (1994), 173-204. MR 98a:58049 Zbl 0798.58021

[Jost 1997] J. Jost, Nonpositive curvature: geometric and analytic aspects, Birkhäuser, Basel, 1997. MR 98g:53070 Zbl 0896.53002

[Jost 1998] J. Jost, Riemannian geometry and geometric analysis, 2nd ed., Springer, Berlin, 1998. Zbl 0997.53500

[Kotani and Sunada 2001] M. Kotani and T. Sunada, "Standard realizations of crystal lattices via harmonic maps", Trans. Amer. Math. Soc. 353:1 (2001), 1-20. MR 2001j:58028 Zbl 0960.58009

[Nikolaev 1995] I. Nikolaev, "The tangent cone of an Aleksandrov space of curvature $\leq K$ ", Manuscripta Math. 86:2 (1995), 137-147. MR 95m:53062 Zbl 0822.53043

[Wang 2000] M.-T. Wang, "Generalized harmonic maps and representations of discrete groups", Comm. Anal. Geom. 8:3 (2000), 545-563. MR 2001m:58039 Zbl 0977.58018

Received July 7, 2006.

\section{JÜRGEN JOST}

Max Planck Institute for Mathematics in the SCiEnCes

INSELSTRASSE 22-26

D-04103 LEIPZIG

GERMANY

jjost@mis.mpg.de

LEONARD TODJIHOUNDE

Institut de Mathématiques et de Sciences Physiques

B.P. 613

PORTO-NOVO

BENIN

leonardt@imsp-uac.org 


\title{
PACIFIC JOURNAL OF MATHEMATICS
}

\author{
http://www.pjmath.org
}

Founded in 1951 by
E. F. Beckenbach (1906-1982)
F. Wolf (1904-1989)

\section{EDITORS}
V. S. Varadarajan (Managing Editor)
Department of Mathematics University of California
Los Angeles, CA 90095-1555
pacific@math.ucla.edu

\author{
Vyjayanthi Chari \\ Department of Mathematics \\ University of California \\ Riverside, CA 92521-0135 \\ chari@math.ucr.edu \\ Robert Finn \\ Department of Mathematics \\ Stanford University \\ Stanford, CA 94305-2125 \\ finn@math.stanford.edu \\ Kefeng Liu \\ Department of Mathematics \\ University of California \\ Los Angeles, CA 90095-1555 \\ liu@math.ucla.edu
}

Paulo Ney de Souza, Production Manager

ACADEMIA SINICA, TAIPEI

CALIFORNIA INST. OF TECHNOLOGY

INST. DE MATEMÁTICA PURA E APLICADA

KEIO UNIVERSITY

MATH. SCIENCES RESEARCH INSTITUTE

NEW MEXICO STATE UNIV.

OREGON STATE UNIV.

PEKING UNIVERSITY

STANFORD UNIVERSITY

\author{
Darren Long \\ Department of Mathematics \\ University of California \\ Santa Barbara, CA 93106-3080 \\ long@math.ucsb.edu \\ Jiang-Hua Lu \\ Department of Mathematics \\ The University of Hong Kong \\ Pokfulam Rd., Hong Kong \\ jhlu@maths.hku.hk \\ Alexander Merkurjev \\ Department of Mathematics \\ University of California \\ Los Angeles, CA 90095-1555 \\ merkurev@math.ucla.edu
}

\section{PRODUCTION}

pacific@math.berkeley.edu

Silvio Levy, Senior Production Editor

Alexandr

\section{SUPPORTING INSTITUTIONS}

UNIVERSIDAD DE LOS ANDES
UNIV. OF ARIZONA
UNIV. OF BRITISH COLUMBIA
UNIV. OF CALIFORNIA, BERKELEY
UNIV. OF CALIFORNIA, DAVIS
UNIV. OF CALIFORNIA, IRVINE
UNIV. OF CALIFORNIA, LOS ANGELES
UNIV. OF CALIFORNIA, RIVERSIDE
UNIV. OF CALIFORNIA, SAN DIEGO
UNIV. OF CALIF., SANTA BARBARA

These supporting institutions contribute to the cost of publication of this Journal, but they are not owners sibility for its contents or policies.

See inside back cover or www.pjmath.org for submission instructions.

Regular subscription rate for 2007: $\$ 425.00$ a year (10 issues). Special rate: $\$ 212.50$ a year to individual $\mathrm{m}$ Subscriptions, requests for back issues from the last three years and changes of subscribers address sh Mathematics, P.O. Box 4163, Berkeley, CA 94704-0163, U.S.A. Prior back issues are obtainable from Main Street, Germantown, NY 12526-5635. The Pacific Journal of Mathematics is indexed by Mathemat PASCAL CNRS Index, Referativnyi Zhurnal, Current Mathematical Publications and the Science Citatio

The Pacific Journal of Mathematics (ISSN 0030-8730) at the University of California, c/o Department Berkeley, CA $94720-3840$ is published monthly except July and August. Periodical rate postage paid at B mailing offices POSTMASTER. send address changes to Pacific Journal of Mathematics. PO Box 4163 


\section{PACIFIC JOURNAL OF MATHEMATICS}

Volume $231 \quad$ No. $2 \quad$ June 2007

The Euclidean rank of Hilbert geometries

OLIVER BLETZ-SIEBERT and THOMAS FOERTSCH

A volumish theorem for the Jones polynomial of alternating knots

279

OLIVER T. DASBACH and XIAO-SONG LIN

On the local Nirenberg problem for the $Q$-curvatures

293

PHILIPPE DELANOË and FrÉDÉRIC ROBERT

Knot colouring polynomials

305

MICHAEL EISERMANN

Some new simple modular Lie superalgebras

Alberto ElduQue

Subfactors from braided $C^{*}$ tensor categories

JULIANA ERLIJMAN and HANS WENZL

An elementary, explicit, proof of the existence of Quot schemes of points

401

Trond Stølen Gustavsen, Dan Laksov and Roy MiKael

SKJELNES

Symplectic energy and Lagrangian intersection under Legendrian

deformations

HAI-LONG HER

Harmonic nets in metric spaces

JÜRGEN JOST and LEONARD TODJIHOUNDE

The quantitative Hopf theorem and filling volume estimates from below

LUOFEI LIU

On the variation of a series on Teichmüller space

GREg McShane

On the geometric and the algebraic rank of graph manifolds 